\title{
Search for First Generation Leptoquarks in ep Collisions at HERA
}

\section{David M. South*i}

Deutsches Elektronen Synchrotron

Notkestrasse 85

22607 Hamburg, Germany

E-mail: david.south@desy.de

\begin{abstract}
A search for first generation scalar and vector leptoquarks produced in $e p$ collisions is performed by the H1 Collaboration at HERA. The full $\mathrm{H} 1$ data sample is used in the analysis, corresponding to an integrated luminosity of $446 \mathrm{pb}^{-1}$. No evidence for the production of leptoquarks is observed in final states with a large transverse momentum electron or with large missing transverse momentum, and constraints on leptoquark models are derived. For leptoquark couplings of electromagnetic strength $\lambda=0.3$, first generation leptoquarks with masses up to $800 \mathrm{GeV}$ are excluded at $95 \%$ confidence level.
\end{abstract}

36th International Conference on High Energy Physics,

July 4-11, 2012

Melbourne, Australia

\footnotetext{
${ }^{*}$ Speaker.

${ }^{\dagger}$ on behalf of the H1 Collaboration.
} 


\section{Leptoquark production at HERA}

The $e p$ collisions at HERA provide a unique possibility to search for new particles coupling directly to a lepton and a quark. Leptoquarks (LQs), colour triplet bosons that do just that, are an example of such particles and appear in many theories attempting to unify the quark and lepton sectors of the Standard Model (SM).

A discussion of the phenomenology of LQs at HERA can be found elsewhere [1]. In the framework of the Buchmüller-Rückl-Wyler (BRW) effective model [2], LQs are classified into 14 types with respect to the quantum numbers spin $J$, weak isospin $I$ and chirality $C$, resulting in seven scalar $(J=0)$ and seven vector $(J=1)$ LQs. Whereas all 14 LQs couple to electron ${ }^{1}$-quark pairs, four of the left-handed LQs, namely $S_{0}^{L}, S_{1}^{L}, V_{0}^{L}$ and $V_{1}^{L}$, may also decay to a neutrino-quark pair. In particular, for $S_{0}^{L}$ and $V_{0}^{L}$ the branching fraction of decays into an electron-quark pair is predicted by the model to be $\beta_{e}=\Gamma_{\mathrm{eq}} /\left(\Gamma_{\mathrm{eq}}+\Gamma_{v_{\mathrm{eq}}}\right)=0.5$, where $\Gamma_{\mathrm{eq}}\left(\Gamma_{v_{\mathrm{eq}}}\right)$ denotes the partial width for the LQ decay to an electron (neutrino) and a quark $q$. The branching fraction of decays into a neutrinoquark pair is then given by $\beta_{v_{e}}=1-\beta_{e}$.

Leptoquarks carry both lepton $(L)$ and baryon $(B)$ quantum numbers, and the fermion number $F=L+3 B$ is assumed to be conserved. Leptoquark processes at HERA proceed directly via $s$ channel resonant LQ production or indirectly via $u$-channel virtual LQ exchange. A dimensionless parameter $\lambda$ defines the coupling at the lepton-quark-LQ vertex. For LQ masses well below the centre-of-mass energy $\sqrt{s}=319 \mathrm{GeV}$, the $s$-channel production of $F=2(F=0) \mathrm{LQs}$ in $e^{-} p\left(e^{+} p\right)$ collisions dominates. However, for LQ masses above $\sqrt{s}$, both the $s$ and $u$-channel processes are important such that both $e^{-} p$ and $e^{+} p$ collisions have similar sensitivity to all LQs types.

The analysis presented here examines LQ decays to a quark and a first generation lepton, following the flavour conservation implicit in the BRW model. In a more general extension of this analysis, dedicated searches have been performed by $\mathrm{H} 1$ for second and third generation leptoquarks, examining final states containing a quark and a charged lepton of a different flavour, i.e. a muon or tau lepton [3,4]. A search for second and third generation leptoquarks was recently performed using the full $\mathrm{H} 1 \mathrm{e} p$ data sample taken at $\sqrt{s}=319 \mathrm{GeV}$, where for a coupling strength of $\lambda=\sqrt{4 \pi \alpha_{\mathrm{em}}}=0.3$, LQs decaying with the same coupling strength to a muon-quark pair or a tau-quark pair are excluded at $95 \%$ confidence level (CL) up to leptoquark masses of $712 \mathrm{GeV}$ and $479 \mathrm{GeV}$, respectively $[4,5]$.

\section{Search for first generation leptoquarks}

This search considers final states where the leptoquark decays into an electron and a quark $e p \rightarrow e X$ or a neutrino and a quark $e p \rightarrow v_{e} X$. The full $\mathrm{H} 1 e p$ data sample has now been analysed [6], which comprises $164 \mathrm{pb}^{-1}$ recorded in $e^{-} p$ collisions and $282 \mathrm{pb}^{-1}$ in $e^{+} p$ collisions, of which $35 \mathrm{pb}^{-1}$ were recorded at $\sqrt{s}=301 \mathrm{GeV}$. Data collected from 2003 onwards were taken with a longitudinally polarised lepton beam. As leptoquarks are chiral particles, these data are analysed in four separate polarisation samples, formed by combining data periods with similar lepton beam charge $e^{ \pm}$and polarisation $P_{e}=\left(N_{R}-N_{L}\right) /\left(N_{R}+N_{L}\right)$, where $N_{R}\left(N_{L}\right)$ is the number of right (left) handed leptons in the beam.

\footnotetext{
${ }^{1}$ Here the term "electron" is used generically to refer to both electrons and positrons, unless otherwise stated.
} 
First generation LQ decays lead to topologies similar to those of deep-inelastic scattering (DIS) neutral current $(\mathrm{NC})$ and charged current $(\mathrm{CC})$ interactions at high negative four-momentum transfer squared $Q^{2}$. The analysis is therefore performed using DIS event selections similar to those used in inclusive DIS analyses [7] and previous first generation LQ searches [8]. Neutral current events are selected by requiring a scattered electron with energy $E_{e^{\prime}}>11 \mathrm{GeV}$ and $Q^{2}>133 \mathrm{GeV}^{2}$ and in the inelasticity region $0.1<y<0.9$. Background from neutral hadrons or photons misidentified as leptons is suppressed by requiring a charged track to be associated to the lepton candidate. Charged current events are selected by requiring significant missing transverse momentum $P_{T}^{\text {miss }}>12 \mathrm{GeV}$, which is due to the undetected neutrino, in the inelasticity region $0.1<y<0.85$. Photoproduction background is suppressed by exploiting the correlation between $P_{T}^{\text {miss }}$ and the ratio $V_{\text {ap }} / V_{\mathrm{p}}$ of transverse energy flow anti-parallel and parallel to the hadronic final state transverse momentum vector [1]. Further details of the event selection can be found in the $\mathrm{H} 1$ publication [6].

A good description of the $\mathrm{H} 1$ data by the SM is observed, where the expectation is dominated by DIS processes in all event samples, with small additional contributions from photoproduction. Mass spectra of the four $\mathrm{H} 1$ data sets taken with a longitudinally polarised lepton beam are shown in figure 1, where both the NC and CC event samples are presented. Since no evidence for LQ production is observed in any of the $\mathrm{NC}$ or CC data samples, the data are used to set constraints on leptoquarks coupling to first generation fermions.

\section{First generation leptoquark limits}

In the absence of a signal, the results of the search are interpreted in terms of exclusion limits on the mass and the LQ coupling. The data are studied in bins in the $M_{L Q}-y$ plane, where the $\mathrm{NC}$ and CC data samples with different lepton beam charge and polarisation are kept as distinct data sets. Limits are determined from a statistical analysis which uses the method of fractional event counting, optimised for the presence of systematic uncertainties. A frequentist analysis is performed of a test statistic obtained from the data for each leptoquark type, mass and coupling hypothesis. A full description of the statistical analysis and limit procedure employed can be found elsewhere [6].

Upper limits on the coupling $\lambda$ obtained at $95 \% \mathrm{CL}$ are shown as a function of the leptoquark mass in figure 2, displayed as groups of scalar and vector LQs for both $F=2$ and $F=0$. For LQ masses near the kinematic limit of $319 \mathrm{GeV}$, the limit corresponding to a resonantly produced LQ turns smoothly into a limit on the virtual effects of both an off-shell $s$-channel LQ process and a $u$-channel LQ exchange. For LQ masses much greater than the HERA centre-of-mass energy the two processes contract to an effective four-fermion interaction. For a coupling of electromagnetic strength $\lambda=\sqrt{4 \pi \alpha_{\mathrm{em}}}=0.3$, LQs produced in ep collisions decaying to an electron-quark or a neutrino-quark pair are excluded at 95\% CL up to leptoquark masses between $277 \mathrm{GeV}\left(V_{0}^{R}\right)$ and $800 \mathrm{GeV}\left(V_{0}^{L}\right)$, depending on the leptoquark type.

Within the framework of the BRW model, the $S_{0}^{L}$ LQ decays to both an electron-quark pair and a neutrino-quark pair, resulting in $\beta_{e}=0.5$. The $\mathrm{H} 1$ limits on $S_{0}^{L}$ are compared to those from other experiments in figure 3, including the similar limit from the ZEUS experiment [9]. The indirect limit from a search for new physics in $e^{+} e^{-}$collisions at LEP by the L3 experiment [10] is also indicated, as well as $\beta_{e}=0.5$ limits from $\mathrm{D} \varnothing$ [11] at the Tevatron and from ATLAS [12] 

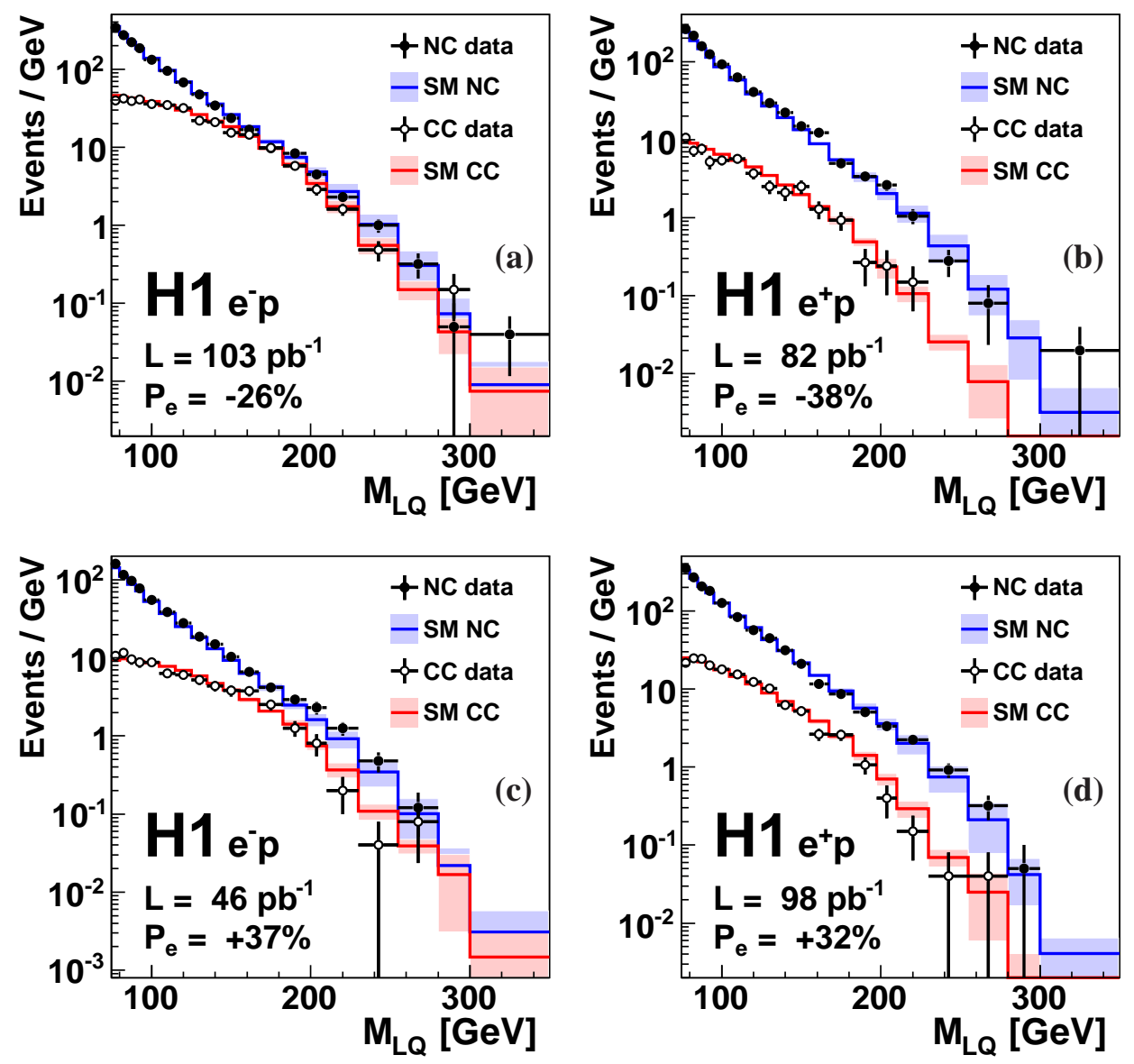

Figure 1: The reconstructed leptoquark mass from the search for first generation leptoquarks using the 2003-2007 H1 data, which was taken with a longitudinally polarised lepton beam. The left-handed electron data (a) and left-handed positron data (b) are shown in the top row; the right-handed electron data (c) and right-handed positron data (d) are shown in the bottom row. The luminosity and average longitudinal lepton polarisation of each data set is indicated. The NC (solid points) and CC (open points) data are compared to the SM predictions (histograms), where the shaded bands indicate the total SM uncertainties.

and CMS [13] at the LHC, based on $\sqrt{s}=7 \mathrm{TeV}$ data taken in 2011. The H1 limits at high leptoquark mass values are also compared to those obtained in a dedicated contact interaction analysis [14]. The additional impact of the CC data can be seen, where a stronger limit is achieved in the LQ analysis, compared to the contact interaction analysis which is based only on NC data. The limits from hadron colliders are based on searches for LQ pair-production and are independent of the coupling $\lambda$, where the strongest current limit for $\beta_{e}=1.0\left(\beta_{e}=0.5\right)$ scalar LQs is $830 \mathrm{GeV}$ $(640 \mathrm{GeV})$ as reported by the CMS collaboration. For a leptoquark mass of $640 \mathrm{GeV}$, this analysis rules out the $S_{0}^{L} \mathrm{LQ}$ for coupling strengths larger than about 0.35 . 

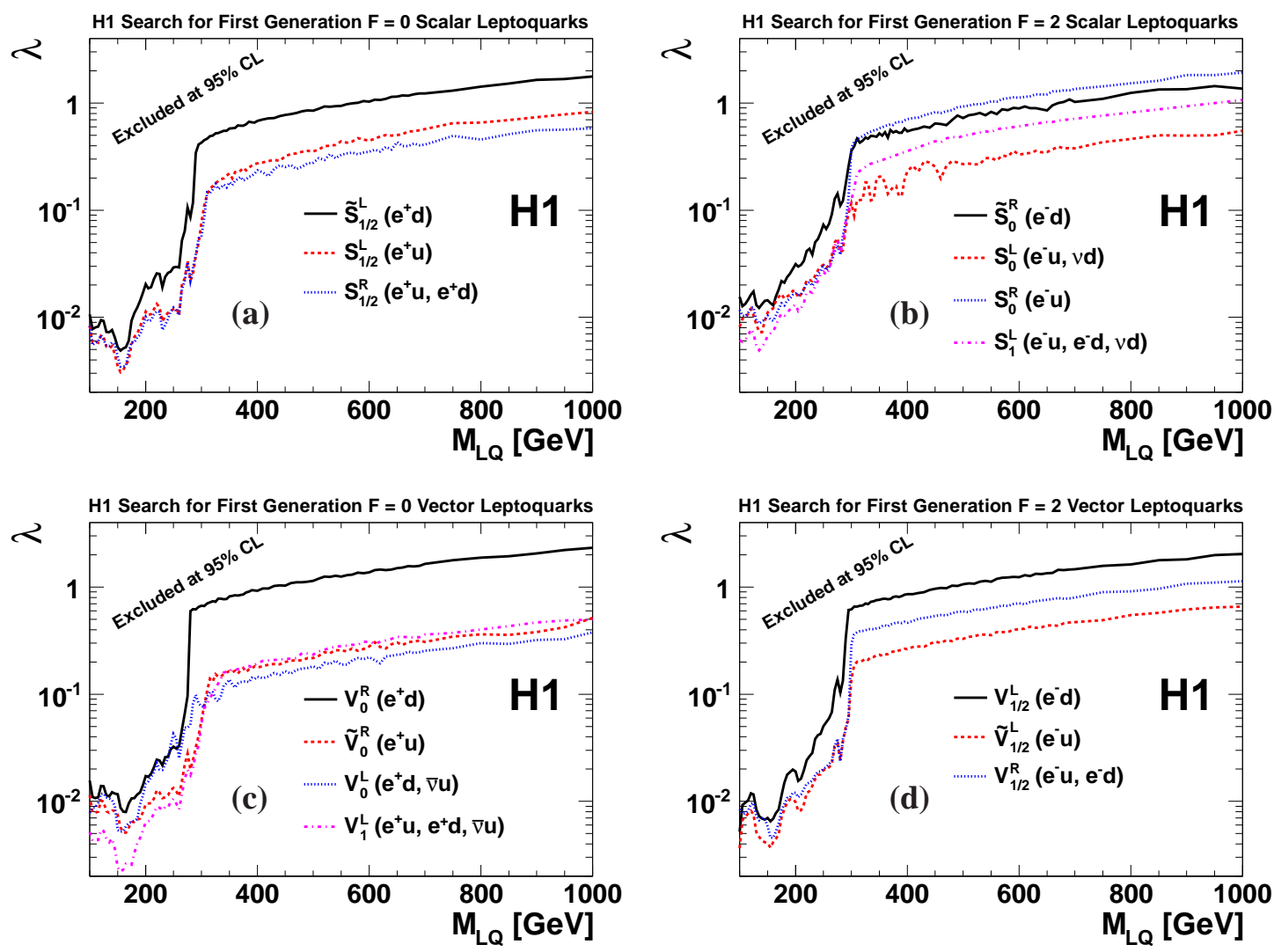

Figure 2: Exclusion limits for the 14 leptoquarks (LQs) described by the Buchmüller, Rückl and Wyler model. The limits are expressed on the coupling $\lambda$ as a function of leptoquark mass for the scalar LQs with (a) $F=0$ and (b) $F=2$ and the vector LQs with (c) $F=0$ and (d) $F=2$. Domains above the curves are excluded at $95 \% \mathrm{CL}$. The parentheses after the LQ name indicate the fermion pairs coupling to the LQ, where pairs involving anti-quarks are not shown.

\section{References}

[1] C. Adloff et al. [H1 Collaboration], A search for leptoquark bosons and lepton flavour violation in $e^{+} p$ collisions at HERA, Eur. Phys. J. C 11 (1999) 447 [Erratum-ibid. 14 (2000) 553]

[hep-ex/9907002].

[2] W. Buchmüller, R. Rückl and D. Wyler, Leptoquarks in lepton-quark collisions, Phys. Lett. B 191 (1987) 442 [Erratum-ibid. 448 (1999) 320].

[3] A. Aktas et al. [H1 Collaboration], Search for lepton flavour violation in ep collisions at HERA, Eur. Phys. J. C 52 (2007) 833 [hep-ex/ 0703004 ].

[4] F. D. Aaron et al. [H1 Collaboration], Search for lepton flavour violation at HERA, Phys. Lett. B 701 (2011) 20 [arXiv:1103.4938].

[5] D. M. South, Search for lepton flavour violation at HERA, in proceedings of 36th International Conference on High Energy Physics, POS ( ICHEP 2012) 140.

[6] F. D. Aaron et al. [H1 Collaboration], Search for first generation leptoquarks in ep collisions at HERA, Phys. Lett. B 704 (2011) 338 [arXiv: 1107 . 3716]. 


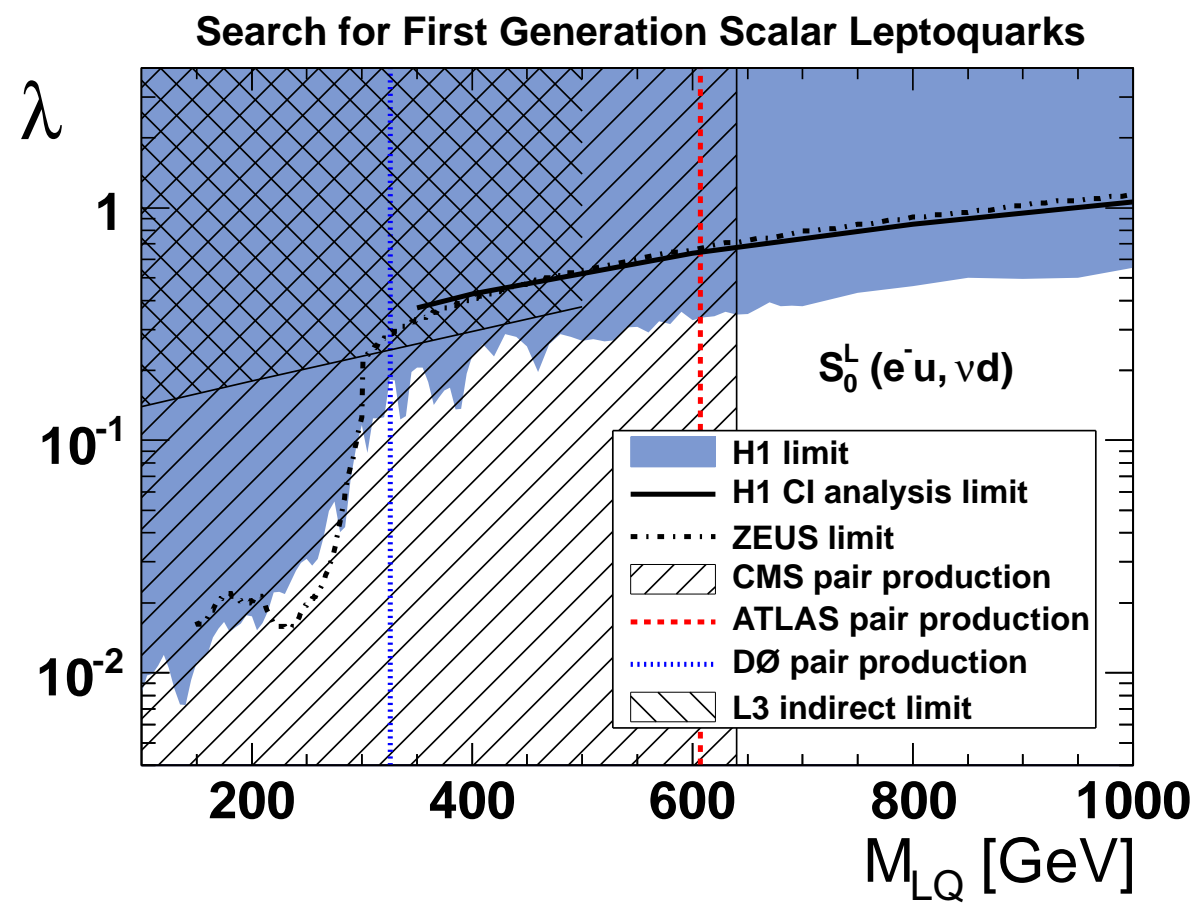

Figure 3: Exclusion limits in the framework of the Buchmüller, Rückl and Wyler model on the coupling $\lambda$ as a function of leptoquark mass for the $S_{0}^{L}$ leptoquark, which has a branching fraction $\beta_{e}=0.5$. Domains above the curves and to the left of the vertical lines are excluded at 95\% CL. Limits from the DØ, L3 and ZEUS experiments and those from the LHC (CMS and ATLAS, $\sqrt{s}=7 \mathrm{TeV}$ data) are shown for comparison, as well as constraints on LQs with masses above $350 \mathrm{GeV}$ from the $\mathrm{H} 1$ contact interaction (CI) analysis.

[7] F. D. Aaron et al. [H1 Collaboration], Inclusive deep inelastic scattering at high $Q^{2}$ with longitudinally polarised lepton beams at HERA, JHEP 1209 (2012) 061 [arXiv: 1206 . 7007].

[8] A. Aktas et al. [H1 Collaboration], Search for leptoquark bosons in ep collisions at HERA, Phys. Lett. B 629 (2005) 9 [hep-ex/ 0506044 ].

[9] H. Abramowicz et al. [ZEUS Collaboration], Search for first-generation leptoquarks at HERA, Phys. Rev. D 86 (2012) 012005 [arXiv: 1205.5179].

[10] M. Acciarri et al. [L3 Collaboration], Search for manifestations of new physics in fermion pair production at LEP, Phys. Lett. B 489 (2000) 81 [hep-ex/0 005028 ].

[11] V. M. Abazov et al. [DØ Collaboration], Search for pair production of first-generation leptoquarks in $p \bar{p}$ collisions at $\sqrt{s}=1.96$ TeV, Phys. Lett. B 681 (2009) 224 [arXiv:0 907.1048 ].

[12] G. Aad et al. [ATLAS Collaboration], Search for first generation scalar leptoquarks in pp collisions at $\sqrt{s}=7$ TeV with the ATLAS detector, Phys. Lett. B 709 (2012) 158 [Erratum-ibid. 711 (2012) 442] [arXiv:1112.4828].

[13] S. Chatrchyan et al. [CMS Collaboration], Search for pair production of first- and second-generation scalar leptoquarks in pp collisions at $\sqrt{s}=7 \mathrm{TeV}$, Phys. Rev. D 86 (2012) 052013 [arXiv:1207.5406].

[14] F. D. Aaron et al. [H1 Collaboration], Search for contact interactions in $e^{ \pm} p$ collisions at HERA, Phys. Lett. B 705 (2011) 52 [arXiv:1107.2478]. 